\title{
Paternal breastfeeding self-efficacy and maternal breastfeeding patterns at three months postpartum
}

\footnotetext{
Seyedeh Zahra Kiaei ${ }^{1}$, Dr. Shahnaz Tork Zahrani ${ }^{2}$, Padideh Jannati ${ }^{3}$, Dr. Maliheh Nasiri ${ }^{4}$

1. Master of Midwifery, Department of Midwifery, School of Nursing and Midwifery, Shahid Beheshti University of Medical Sciences, Tehran, Iran.

2. Ph.D. in Midwifery, Department of Midwifery, School of Nursing and Midwifery, Shahid Beheshti University of Medical Sciences, Tehran, Iran.

3. Master of Midwifery, Department of Midwifery, School of Nursing and Midwifery, Shahid Beheshti University of Medical Sciences, Tehran, Iran.

4. Ph.D. in Biostatistics, Assistant Professor, Department of Basic Sciences, School of Nursing and Midwifery, Shahid Beheshti University of Medical Sciences, Tehran, Iran.
}

Abstract

Introduction: Evidence suggests that breastfeeding has beneficial effects on both mother and baby. Fathers' attitudes and self-efficacy are effective factors in breastfeeding. Paternal breastfeeding self-efficacy means the ability of fathers to help their spouses to breastfeed their infants, which seems to affect breastfeeding. The aim of this study was to evaluate the self-efficacy of fathers and its relationship with infant feeding patterns at three months.

Methods: This descriptive correlational study was conducted in 2019 on 206 fathers during the discharge of their wife after childbirth. The inclusion criteria were having a singleton infant, birth weight of 2500-4000 g, Apgar score of 8-10, willingness to participate in the study, the parents living together, and not taking any drugs affecting breastfeeding. A demographic information questionnaire and the paternal breastfeeding self-efficacy scale were completed by the fathers; the maternal breastfeeding pattern questionnaire was also completed three months after delivery. Data were analyzed in SPSS-23 statistical software using the independent t-test, Spearman's test, Pearson's coefficient, the ANOVA, and the Chi-square test.

Findings: The paternal breastfeeding self-efficacy score was 58.33 in this study. The paternal breastfeeding self-efficacy score of the fathers attending antenatal classes was higher than the others $(P$-value $=0.003)$. There was a significant relationship between paternal cooperation in prenatal care and paternal breastfeeding self-efficacy score $(P$-value $=0.001)$. There was no correlation between paternal breastfeeding self-efficacy scores and maternal breastfeeding patterns three months after delivery ( $P$-value=0.99).

Conclusion: According to the results, there was no relationship between paternal breastfeeding self-efficacy scores and maternal breastfeeding patterns three months after delivery. However, significant relationships were found between paternal breastfeeding self-efficacy score at discharge and the fathers' cooperation in prenatal care and attendance of antenatal classes.

Keywords: Self-Efficacy, Paternal Breastfeeding Self-Efficacy, Maternal Breastfeeding Patterns 


\section{Introduction}

Evidence suggests that breastfeeding has beneficial effects on both the mother and newborn and plays a significant role in controlling chronic diseases (1). Statistics show that the lack of breastfeeding in developing countries is leading to the death of 1 child every 30 seconds and 1.5 million children per year, accounting for $70 \%$ of the neonatal mortality in these countries (2). Factors such as the mother's age and education, family income, family support, prenatal measures, decisions about the first breastfeeding, time of the first feeding, and maternal skills and selfefficacy are effective in successful breastfeeding (3).

Self-efficacy or the perceived ability to successfully perform a task is an important principle linking awareness to action (4). Individuals' beliefs about their abilities affect the action they desire to take, their efforts to take that action, and how they resist hardships on the path. Individuals who lack self-efficacy are not able to effectively control situations even when they possess the necessary knowledge and skills (5).

Fathers' attitude and their support are alterable factors that significantly affect breastfeeding onset (6). Paternal breastfeeding self-efficacy denotes the father's ability to help his wife breastfeed their newborn (7). Breastfeeding cessation may be due to fathers' feelings of frustration and guilt or even helplessness as they might think that they cannot help, and starting formula feeding might look like the easiest solution (8).

The involvement of fathers in breastfeeding training can improve the amount and duration of exclusive breastfeeding (9) (10). Fathers' participation in breastfeeding promotion programs in maternity wards can significantly increase EBF in the first six months of a newborn's life, probably due to the information given to fathers in these wards about EBF.

Paternal training can encourage fathers and thus have a positive effect on the mothers in avoiding feeding the infant with water, herbal drinks, or different types of formula (11). Fathers' self-efficacy score is significantly correlated with mothers' self-efficacy, fathers' attitude toward breastfeeding, the importance of breastfeeding for the father, the promotion of breastfeeding, EBF, and the belief in making progress in breastfeeding (7).

Improved paternal breastfeeding self-efficacy and mothers' perception of fathers' cooperation and assistance show that programs to support parental cooperation in breastfeeding can be beneficial for the parents (12).

Given the WHO recommendations about the role of breastfeeding in children's health, the impact of parents' self-confidence in this matter, and the more pronounced role of fathers and their breastfeeding support, there is certainly a need to conduct further studies on this subject. Therefore, this research seeks to determine the relationship between paternal breastfeeding selfefficacy and maternal breastfeeding patterns. 


\section{Methods}

This descriptive-correlational study was conducted after obtaining the necessary approvals, including from the ethics committee of the university (code: IR.SBMU.PHARMACY.REC.1398.060). After receiving the authorities' permission and presenting to the postpartum ward of Alborz Hospital in Karaj, the researcher selected samples from the fathers who were present in the ward their wife's discharge after delivery. The sample size was estimated as 164 using the sample size estimation formula and considering a type-I error of $\alpha=0.05$, but to take account of a potential $10 \%$ sample loss and based on the research team's opinion, the sample size was increased to 220. The correlation between paternal breastfeeding self-efficacy and maternal feeding patterns three months after delivery was estimated as $r=0.25$.

$$
\begin{gathered}
n \geq\left[\frac{\left(z_{1-\alpha / 2}+z_{1-\beta}\right)}{0.5 \times \ln [(1+r) /(1-r)]}\right]^{2}+3 \\
r=0.25 \\
\alpha=0.05 \Rightarrow z_{1-\alpha / 2}=1.96 \\
\beta=0.10 \Rightarrow z_{1-\beta}=1.28
\end{gathered}
$$

The samples entered the study after giving their verbal consent to participate and getting briefed on the study objectives and ensured about the confidentiality of their data. The inclusion criteria were willingness to participate in the study, the parents living together, not taking any drugs affecting breastfeeding, singleton birth, a birth weight over 2500, a gestational age of 37 weeks or more at delivery, a normal Apgar score at birth, and no neonatal diseases or anomalies. The exclusion criteria were lack of access to the subjects to complete the questionnaire three months after delivery, participants' unwillingness to continue cooperation, and serious postpartum complications for the mother or newborn that had either separated them or led to the death of any one of them. The Breastfeeding Self-Efficacy Scale-Short Form (7) was used to assess paternal self-efficacy. This questionnaire consists of 14 items scored based on a 5-point Likert scale from Always confident to Not at all confident. The lowest score is 14 and the highest 70 . This scale was originally developed in English and was translated into Persian using the forward-backward translation method. For this purpose, the English version of the scale was first translated into Persian by two translators with expertise in medical texts. Next, the translations were compared by two obstetricians who were also faculty members, and a Persian version of the tool was prepared by matching the items in terms of meaning and concept and selecting the best options. To ensure the expressiveness of the sentences in the Persian text and their match with the original text, the initial translated version was re-translated into English by two other translators fluent in English who had not previously seen the original questionnaire. Then, by comparing the two English versions and making the necessary corrections, a single version of the English questionnaire was developed (13). To assess the questionnaire's face validity, it was distributed among ten fathers, who were asked to comment on the legibility, clearness, writing style, grammar 
and spelling of the words, and the ease of completing the tool. In the present study, the reliability of this questionnaire was assessed by calculating its Cronbach alpha coefficient, which was 0.94. The newborns' nutrition was assessed by the infant nutrition form three months after delivery via phone calls to the mothers participating in the study. This form was prepared using the WHO model and was distributed among ten faculty members of the Midwifery and Reproductive Health Department of Shahid Beheshti University of Medical Sciences for content and face validity evaluation. The final infant nutrition form was developed based on their comments. A demographic information questionnaire was also designed for the study and its content validity was approved. Data were analyzed in SPSS-23 software using descriptive statistical methods, including tables of frequency, percentage, mean and standard deviation, as well as inferential statistical methods, including the independent t-test, ANOVA, Spearman's test, and the Chisquare test.

At the end of sampling, 14 people were excluded from the study, reducing the sample size to 206. The reasons for this sample loss included not answering the phone calls $(n=10)$, the mother's unwillingness to continue cooperation (7), and the death of the infant (7).

\section{Results}

In the present study, the mean age of the fathers was 33.79 years. Table 1 presents the fathers' demographic characteristics and Table 2 presents the infants' characteristics along with the maternal breastfeeding patterns. Among the fathers participating in the study, $22.8 \%$ had participated in antenatal classes and $67.6 \%$ had cooperated in prenatal care. Also, $87.9 \%$ of the fathers chose breastfeeding as their preferred form of feeding for their infant during their wife's

discharge from the postpartum ward. Also, $85.9 \%$ of the mothers reported having received adequate paternal breastfeeding support during the three months after the delivery, while $2.4 \%$ of the fathers had opposed breastfeeding during the three months after delivery. Among the reasons for their opposition were thinking that breast milk was not sufficient, being concerned about the mother's comfort, and being concerned about the deformation of the mother's breasts due to breastfeeding. Table 3 examines the relationship between the paternal self-efficacy score and the examined variables, indicating that only two variables, namely the father's cooperation in prenatal care $(P=0.0001)$ and the father's attendance of antenatal classes $(P=0.0003)$, were correlated with the paternal self-efficacy score. Table 4 shows the relationship between maternal breastfeeding patterns and the study variables. The father's support for the mother in breastfeeding was the only variable that correlated with the breastfeeding pattern $(P=0.0006)$.

\section{Table 1. The absolute and relative frequency distribution of the study subjects based on the fathers' demographic characteristics}




\begin{tabular}{|c|c|c|c|}
\hline \multicolumn{2}{|c|}{$\begin{array}{ll}\text { Variable } & \text { Frequency } \\
\end{array}$} & Number & Percentage \\
\hline \multirow{9}{*}{$\begin{array}{l}\text { Age group } \\
\text { (Year) }\end{array}$} & $22-26$ & 13 & 6.31 \\
\hline & $27-31$ & 61 & 29.61 \\
\hline & $32-36$ & 77 & 37.38 \\
\hline & $37-41$ & 40 & 19.42 \\
\hline & $42-46$ & 11 & 5.34 \\
\hline & $47-51$ & 2 & 0.97 \\
\hline & $52-56$ & 2 & 0.97 \\
\hline & Total & 206 & 100 \\
\hline & Mean \pm SD & \multicolumn{2}{|c|}{$33.5 \pm 79.27$} \\
\hline \multirow{7}{*}{ Education } & Primary school & 54 & 26.2 \\
\hline & High school & 109 & 52.9 \\
\hline & Associate degree & 15 & 7.3 \\
\hline & Bachelor's degree & 19 & 9.2 \\
\hline & Master's degree & 7 & 3.4 \\
\hline & Ph.D. & 2 & 1 \\
\hline & Total & 206 & 100 \\
\hline \multirow{4}{*}{ Father's occupation } & Unemployed & 2 & 1 \\
\hline & Employed & 44 & 21.4 \\
\hline & Self-employed & 160 & 77.7 \\
\hline & Total & 206 & 100 \\
\hline \multirow{3}{*}{ Housing status } & Renting & 103 & 50 \\
\hline & Owner & 103 & 50 \\
\hline & Total & 206 & 100 \\
\hline \multirow{3}{*}{ Father's income status } & Less than sufficient & 104 & 4.4 \\
\hline & Sufficient & 94 & 50 \\
\hline & To the extent of saving & 8 & 45.6 \\
\hline
\end{tabular}




\begin{tabular}{|c|c|c|c|}
\hline \multirow{4}{*}{ Drug use status } & Total & 206 & 100 \\
\hline \multirow{3}{*}{$*$} & Smoking & 34 & 16.5 \\
\cline { 2 - 4 } & Alcohol & 3 & 1.5 \\
\cline { 2 - 4 } & None & 169 & 82 \\
\cline { 2 - 4 } & Total & 206 & 100 \\
\hline
\end{tabular}

Table 2. The absolute and relative frequency distribution of the newborns' gender and type of delivery in the studied mothers

\begin{tabular}{|c|c|c|c|}
\hline \multicolumn{2}{|l|}{ Variable } & Number & Percentage \\
\hline \multirow{3}{*}{ Newborn's gender } & Female & 104 & 50.5 \\
\cline { 2 - 4 } & Male & 102 & 49.5 \\
\cline { 2 - 4 } & Total & 206 & 100 \\
\hline \multirow{3}{*}{ Type of delivery } & Vaginal delivery & 103 & 50 \\
\cline { 2 - 4 } & Cesarean section & 103 & 50 \\
\cline { 2 - 4 } & Total & 206 & 100 \\
\hline
\end{tabular}

Table 3. The relationship between paternal breastfeeding self-efficacy scores and the study variables

\begin{tabular}{|c|c|c|}
\hline Variable & Test & P-value \\
\hline Father's age & Pearson & 0.949 \\
\hline Occupation & ANOVA & 0.822 \\
\hline Education & ANOVA & 0.731 \\
\hline Father's cooperation in prenatal care & Independent-t & 0.001 \\
\hline Father's attendance of antenatal classes & Independent-t & 0.003 \\
\hline Father's decision on the type of neonatal feeding & ANOVA & 0.347 \\
\hline Father's income status & ANOVA & 0.634 \\
\hline Father's occupation & ANOVA & 0.822 \\
\hline Breastfeeding patterns three months after delivery & ANOVA & 0.99 \\
\hline Father's support for breastfeeding & ANOVA & 0.757 \\
\hline
\end{tabular}


Table 4. The relationship between the paternal variables and maternal breastfeeding patterns

\begin{tabular}{|c|c|}
\hline Variable & Chi-square test results \\
\hline Father's education & 0.240 \\
\hline Father's occupation & 0.194 \\
\hline Father's income status & 0.406 \\
\hline Father's attendance of antenatal classes & 0.203 \\
\hline Father's cooperation in prenatal care & 0.180 \\
\hline Housing status & 0.812 \\
\hline Father's decision on the type of neonatal feeding & 0.279 \\
\hline Father's support of the mother for breastfeeding & 0.006 \\
\hline
\end{tabular}

\section{Discussion}

This study was conducted to determine paternal breastfeeding self-efficacy and its relationship with maternal breastfeeding patterns. The mean paternal breastfeeding self-efficacy score before the discharge of the mothers was 58.33 out of 70 in the present study. Since this study is the first on the breastfeeding self-efficacy of Iranian fathers, there was no other information that could be used for comparison purposes. In a study by Dennis, the self-efficacy score of Canadian fathers was 48.96. This discrepancy and the higher self-efficacy score of Iranian fathers can be due to the cultural differences between the two samples and the importance given to breastfeeding upon birth in Iranian families. Meanwhile, no relationship was found between paternal breastfeeding self-efficacy and maternal breastfeeding patterns three months after delivery in this study. This finding contradicts the results reported by Dennis, who found a significant relationship between paternal self-efficacy scores, attitudes toward breastfeeding, breastfeeding rate, and EBF (7). Nonetheless, Dennis' study did not specify the pattern of breastfeeding at 12 weeks (14) and the rate of EBF. In the present study, only 5.8\% of Iranian children used formula feeding at three months of age and $74.3 \%$ were exclusively breastfed, and there was no relationship between this type of breastfeeding and paternal self-efficacy. In another study, (15) reported a relationship between paternal self-efficacy score and breastfeeding support and the onset of breastfeeding, but the long-term breastfeeding pattern (12) was not investigated in the study. Several factors affect maternal breastfeeding patterns, including but not limited to the role of the father. Research findings indicate a direct relationship between fathers' support for breastfeeding and the greater 
likelihood of continued breastfeeding (16). In contrast, some studies do not find the role of fathers significant in EBF. A study by (17)in India suggested that although fathers' attitude toward breastfeeding is effective, it does not affect the duration of EBF. Meanwhile, the present study only examined paternal breastfeeding self-efficacy in relation to breastfeeding patterns. One of the limitations of the present study was the impossibility of measuring the self-efficacy of the fathers three months after delivery. If the self-efficacy of the fathers had been measured three months after delivery, the trends of change in this variable could have been better evaluated and analyzed.

According to the results, there was a significant relationship between paternal breastfeeding selfefficacy scores after childbirth and the fathers' cooperation in prenatal care; the mean paternal breastfeeding self-efficacy score was higher in the group of fathers who cooperated in prenatal care $(P$-value $=0.001)$. There was also a significant relationship between paternal breastfeeding self-efficacy scores and their attendance of antenatal classes ( $P$-value=0.003). (18) found that fathers' attendance of antenatal classes makes them more prepared for accepting the role of father. Studies have also shown that educating fathers before and after childbirth can improve EBF at six and four months postpartum and reduce the likelihood of formula feeding at two months postpartum and the incidence of breastfeeding problems (17).

In the present study, there was no correlation between paternal breastfeeding self-efficacy scores at discharge and the fathers' decision on the type of neonatal feeding after childbirth and their income status, occupation, age, education, and breastfeeding support.

Fathers' support is a modifiable and alterable factor. Parental cooperation in breastfeeding offers an ideal framework for designing interventions to increase fathers' involvement and helping the parents work together to meet their breastfeeding goals (16). In the present study, a significant relationship was observed between the fathers' support for breastfeeding in the three months after delivery and maternal breastfeeding patterns at three months postpartum ( $P$-value=0.006). This finding is consistent with the results of another study (10), although no relationship was observed between maternal breastfeeding patterns three months after delivery and occupation, education, income, housing status, father's attendance of antenatal classes, father's cooperation in prenatal care, and father's decision on the type of neonatal feeding at the time of discharge. (19) found that fathers' attitudes were effective in supporting breastfeeding but were not correlated with the duration of EBF.

\section{Conclusion}

According to the present findings, no correlation was found between paternal breastfeeding selfefficacy scores and maternal feeding patterns at three months postpartum. Paternal breastfeeding self-efficacy scores correlated significantly with the father's attendance of antenatal classes, and the father's cooperation in prenatal care. Nonetheless, there was no significant relationship 
between the fathers' age, occupation, education, income, and support for breastfeeding and the paternal breastfeeding self-efficacy score. Furthermore, there was no relationship in this study between maternal breastfeeding patterns and the father's education, father's occupation, income status, housing status, and the father's decision on the type of neonatal feeding. Meanwhile, there was a significant relationship between maternal breastfeeding patterns and paternal support for breastfeeding (P-value=0.006).

\section{Declaration}

\section{Ethical approval}

This study was conducted after obtaining the code of ethics from the ethics committee (IR.SBMU.PHARMACY.REC.1398.060) and a written letter of introduction from Shahid Beheshti University of Medical Sciences and receiving permission from Alborz University of Medical Sciences.

\section{consent to participate}

informed consent was obtained from the participants.

\section{Consent for publication}

This study is not restricted for publication.

\section{Availability of data and material}

The data set created or analyzed during the current study will be provided at the request of the relevant author.

\section{Competing interests}

There is no conflict of interest in this study.

\section{Funding}

There are no financial benefits in this study.

\section{Authors' contributions}

Seyedeh Zahra Kiaie and Shahnaz Tork Zahrani designed the research. Seyedeh Zahra Kiaie wrote the manuscript with the support of Padideh Jannati and Shahnaz Tork Zahrani. And Maliha Nasiri analyzed the data. Shahnaz Tork Zahrani and Padideh Jannati helped oversee the project. Seyedeh Zahra Kiaie founded the main idea.

\section{Acknowledgments}

This article was extracted from the M.Sc. dissertation of Ms. Seyedeh Zahra Kiaie in midwifery, supervised by Dr. Shahnaz Tork Zahrani, at Shahid Beheshti University of Medical Sciences and Health Services. Hereby, the researchers wish to express their gratitude to the Research Deputy of 
Shahid Beheshti University of Medical Sciences and Health Services and the esteemed officials of Alborz Hospital, especially the staff of the postpartum ward, who assisted in conducting this research.

.) Binns C, Lee M, Low WY. The Long-Term Public Health Benefits of Breastfeeding. Asia Pac J Public Health. 2016;28(1):7-14.

.r VARAEI S, MEHRDAD N, BAHRANI N. THE RELATIONSHIP BETWEEN SELF-EFFICACY AND BREASTFEEDING, TEHRAN, IRAN. HAYAT. 2009;15--:( $($ )

.r Krouse AM. The Family Management of Breastfeeding Low Birth Weight Infants. Journal of Human Lactation. 2002;18(2):155-65.

. $\quad$ Taghdisi MH, Latifi M, Afkari ME, Dastoorpour M, Estebsari F, Jamalzadeh F. The Impact of Educational Intervention to Increase Self Efficacy and Awareness for the Prevention of Domestic Violence Against Women. Iranian Journal of Health Education and Health Promotion. 2015;3(1):32-8.

.$\quad$ Bandura A. Perceived self-efficacy in the exercise of control over AIDS infection. Evaluation and Program Planning. 1990;13(1):9-17.

$.7 \quad$ Wolfberg AJ, Michels KB, Shields W, O'Campo P, Bronner Y, Bienstock J. Dads as breastfeeding advocates: results from a randomized controlled trial of an educational intervention. Am J Obstet Gynecol. 2004;191(3):708. IT-

.V Dennis CL, Brennenstuhl S, Abbass-Dick J. Measuring paternal breastfeeding self-efficacy: A psychometric evaluation of the Breastfeeding Self-Efficacy Scale-Short Form among fathers. Midwifery. 2018;64:17-22.

.$\wedge \quad$ Sherriff N, Hall V. Engaging and supporting fathers to promote breastfeeding: a new role for Health Visitors? Scand J Caring Sci. 2011;25(3):467-75.

.9 Boccolini CS, Boccolini PdMM, Monteiro FR, Venâncio SI, Giugliani ERJ. Breastfeeding indicators trends in Brazil for three decades. Rev Saude Publica. 2017;51:108.-

.1. Su M, Ouyang YQ. Father's Role in Breastfeeding Promotion: Lessons from a Quasi-Experimental Trial in China. Breastfeeding medicine : the official journal of the Academy of Breastfeeding Medicine. 2016;11:144-9.

.11 Susin LR, Giugliani ER. Inclusion of fathers in an intervention to promote breastfeeding: impact on breastfeeding rates. J Hum Lact. 2008;24(4):386-92; quiz 451-3.

ir Abbass-Dick J, Stern SB, Nelson LE, Watson W, Dennis CL. Coparenting breastfeeding support and exclusive breastfeeding: a randomized controlled trial. Pediatrics. 2015;135(1):102-10.

. Ir Pashandi Sh., Khaghanizade M., Ebadi A. Review of translation and cultural adaptation process of questionnaires. Bimonthly of Education Strategies in Medical Sciences. 2009;2(3):117-20.

. I Brandao S, Mendonca D, Dias CC, Pinto TM, Dennis CL, Figueiredo B. The breastfeeding selfefficacy scale-short form: Psychometric characteristics in Portuguese pregnant women. Midwifery. 2018;66:49-55.

.10 Carlisle SA. Fathers and Breastfeeding: The Role of Paternal Breastfeeding Support Self-Efficacy in Breastfeeding Initiation: University of Akron; 2013. 
.17 Abbass-Dick J, Dennis CL. Breast-feeding Coparenting Framework: A New Framework to Improve Breast-feeding Duration and Exclusivity. Family \& community health. 2017;40(1):28-31.

.IV Mahesh PKB, Gunathunga MW, Arnold SM, Jayasinghe C, Pathirana S, Makarim MF, et al. Effectiveness of targeting fathers for breastfeeding promotion: systematic review and meta-analysis. BMC Public Health. 2018;18(1):1140.

. 1 Premberg A, Lundgren I. Fathers' Experiences of Childbirth Education. J Perinat Educ. 2006;15(2):21-8.

.19 Karande S, Perkar S. Do fathers' attitudes support breastfeeding? A cross-sectional questionnairebased study in Mumbai, India. Indian journal of medical sciences. 2012;66(1-2):30-9. 